ISSN 1991-8631

Original Paper

http://indexmedicus.afro.who.int

\title{
Caractérisation des sols de la zone des Niayes de Pikine et de Saint Louis (Sénégal)
}

\author{
Ousmane NDIAYE ${ }^{1}$, Aly DIALLO ${ }^{1}$, François MATTY ${ }^{3}$, Amath THIAW ${ }^{1}$, \\ Rokhaya Daba FALL ${ }^{2}$ et Aliou GUISSE ${ }^{1,4}$ * \\ ${ }^{I}$ Département de Biologie Végétale, Faculté des Sciences et Techniques, Université Cheikh Anta Diop de \\ Dakar, B.P. 5005 Dakar-Fann (Sénégal). \\ ${ }^{2}$ Institut National de Pédologie, Hann Mariste BP 6225, \\ ${ }^{3}$ Institut des Sciences de l'Environnement adresse (ISE), FST, UCAD BP 5005 Dakar. \\ ${ }^{4}$ Observatoire Homme Milieu Tessékére. \\ *Auteur correspondant,E-mail: alguisse@orange.sn,aliou.guisse@ucad.edu.sn ; \\ Tel. $(+221) 776389669$
}

\section{RESUME}

La zone des Niayes fournit l'essentiel de la production maraîchère du Sénégal. Cependant, son potentiel agricole a été sérieusement réduit par la dégradation des terres dont les aléas climatiques de ces dernières décennies ainsi que les mauvaises pratiques culturales sont les causes. Cette étude se propose de caractériser les sols de la zone afin d'identifier les différentes unités. Pour se faire, des profils pédologiques ont été décrits dans différentes localités, et leurs paramètres physico-chimiques déterminés. Les résultats de l'analyse factorielle de correspondance (AFC) de la matrice des profils et paramètres physico-chimiques ont révélé l'existence, selon la salinité, de trois groupes de sols répartis dans toutes les Niayes. L'analyse a aussi montré l'existence selon l'alcalinité ou l'acidité et la teneur en calcium échangeable, de deux groupes de sols retrouvés uniquement dans la partie nord. La salinisation des sols plus marquée au niveau des bas-fonds de la partie sud, s'expliquerait par l'abaissement de la nappe phréatique et l'intrusion des eaux de mer. A cela s'ajoute les apports non négligeables des embruns marins dans les zones proches de l'océan, et les effets du barrage de Diama et du canal de délestage ouvert sur la Langue de Barbarie à Saint-Louis.

(C) 2012 International Formulae Group. All rights reserved.

Mots clés : AFC, Niayes, Profil pédologique.

\section{INTRODUCTION}

Les Niayes constituent une zone agroécologique d'une importance capitale dans l'économie du Sénégal. Cette région se caractérise par des conditions physiques (climat doux et humide, faible profondeur de la nappe phréatique, typologie des sols, etc.) et un contexte socio-économique favorables à des activités agro-pastorales intenses. Elle représente la principale zone de cultures maraîchères du Sénégal et offre de bonnes conditions pour l'arboriculture (Ba, 2008). Le taux d'exploitation agricole des Niayes (65\%) est le plus important au niveau national, après celui du bassin arachidier (Khouma, 1998).

Cependant, cette zone connaît d'intenses phénomènes d'érosion éolienne dus aux 
mouvements des dunes maritimes et semi continentales. Il s'en est suivi une avancée des dunes vers les cuvettes qui a sérieusement réduit leur potentiel agricole (Fall et al., 2001). De plus, la sécheresse qui sévit dans la zone soudano-sahélienne depuis plus de trois décennies est fortement ressentie dans les Niayes. Ce phénomène se traduit par un déficit pluviométrique qui est à l'origine de l'abaissement de la nappe phréatique et de la remontée du biseau salé.

Ces situations ont engendré une dégradation du couvert végétal avec des effets néfastes sur les sols et les systèmes traditionnels de production. Cette dégradation progressive des écosystèmes est accentuée par l'action anthropique à travers des pratiques agricoles inappropriées, mais aussi par l'expansion démographique et l'urbanisation accrue.

Plusieurs stratégies (reboisement, phosphatage, mis en défens, etc.) ont été mises en œuvre pour la reconstitution de ces écosystèmes naturels. Selon Trochain (1940), la mise en défens de la région est l'une des actions les plus efficaces pour préserver ses ressources. Cependant, ces stratégies n'ont pas conduit à des résultats escomptés car n'ayant pas eu comme préalable la connaissance de l'état actuel des ressources (Diallo, 2006). De plus, la mise en défens pourrait compromettre les potentialités économiques de la zone. Ainsi, il serait intéressant pour mieux comprendre les contraintes de ces écosystèmes fragiles, de procéder à leur caractérisation écologique.

L'objectif de ce travail est de caractériser la zone des Niayes sur le plan pédologique afin de diagnostiquer les processus de dégradation des sols et d'apporter des informations nécessaires pour la reconstitution et la gestion durable des ressources naturelles de cette région.

\section{MATERIEL ET METHODES}

\section{La zone d'étude}

Située le long du littoral nord sénégalais appelé communément grande côte, la zone des Niayes s'étend de Dakar à SaintLouis, dans une bande de $180 \mathrm{~km}$ de long sur 5 à $30 \mathrm{~km}$ de large (Fall et al., 2001). Sur le plan administratif, elle couvre une partie des régions de Saint-Louis et de Louga, les départements de Tivaouane et Thiès et toute la région de Dakar (Figure 1).

Sur le plan géomorphologique, les Niayes sont formées d'une série de dunes qui alternent avec des cuvettes inter-dunaires. Selon l'origine du matériel, les conditions et l'époque de sa mise en place, nous distinguons les systèmes dunaires littoraux constitués de dunes blanches encore appelés dunes vives et de dunes jaunes ou dunes semifixées et un système dunaire continental. Entre les dunes jaunes semi-fixées et les dunes rouges fixées, apparaissent les cuvettes interdunaires utilisées pour les cultures maraîchères et dont la survie est étroitement liée à la fixation des dunes maritimes (Dia, 1992).

Selon Fall et al. (2001), les systèmes dunaires littoraux sont caractérisés par des plages de sable coquillier constamment repris par le vent et dont leur origine remonte du subactuel à l'actuel (2 000 à 1800 ans avant l'Actuel), tandis que les dunes rouges continentales constituées de sols rouges ou « diors » dans la terminologie locale, auraient été mises en place depuis l'ogolien (15000 à 20000 ans avant l'Actuel).

Le climat est de type tropical caractérisé par deux saisons principales: une saison des pluies, courte s'étendant de Juillet à Octobre et une saison sèche plus longue subdivisée en sous saisons (Figure 2).

Les sols varient du nord au sud. Leur nature et leur distribution s'articulent autour des grands ensembles géomorphologiques présents sur le territoire. On distingue ainsi six types de sols (Fall et al., 2001): les sols minéraux bruts d'apport qui caractérisent les dunes vives dans lesquelles l'évolution des sols y est pratiquement nulle; les sols ferrugineux tropicaux peu lessivés qui caractérisent les dunes rouges et occupant près 
de $70 \%$ de la région des Niayes; les sols brunrouges iso-humiques que l'on rencontre dans la partie nord-ouest de Louga et sud-ouest de Saint-Louis; les vertisols rencontrés dans les dépressions à argile gonflante, localisées dans la zone de Sébikotane (plateau de Bargny), sur l'axe Somone et le lac Tanma; les sols halomorphes caractérisés par leur salinité et/ou alcalinité, situés aux environs des lagunes côtières barrées par les cordons dunaires et les sols hydromorphes des dépressions très favorables au développement du maraîchage.

Sur le plan hydrologique, les ressources en eau des Niayes proviennent essentiellement de la nappe phréatique des sables quaternaires. Ce potentiel souffre d'une surexploitation qui se manifeste par un rabattement progressif en profondeur de la nappe.

Deux sites ont été choisis comme base d'échantillonnage; il s'agit de la communauté rurale de Gandon (région de Saint-Louis) et la commune de Pikine (Figure 3).

Le choix des sites s'explique par l'existence d'un gradient climatique entre Pikine (au Sud) et Saint-Louis (au Nord) qui serait à l'origine de deux sous zones écogéographiques (Anonyme, 1990): une région faiblement aride au sud et une région franchement aride au nord. Ce choix se justifie également par la diversité des types de sols au niveau de ces deux sites: dans la partie méridionale des Niayes les sols hydromorphes et les sols ferrugineux tropicaux peu lessivés sont prédominants, tandis que dans la partie septentrionale les sols brun-rouges iso-humiques sont plus importants.

\section{Collecte de données}

L'échantillonnage consiste à positionner des profils pédologiques représentatifs des différentes unités de sols identifiées grâce à la carte morphopédologique du Sénégal au 1/500.000. Ainsi, dans chaque site d'étude retenu, d'autres sites secondaires ont été choisis et un profil a été réalisé dans chacun d'eux, soit trois profils dans les Niayes de Pikine et sept dans les Niayes de Saint-Louis (trois dans la partie éloignée de l'océan et quatre dans la zone proche de l'océan).

\section{Analyses au laboratoire}

Pour la caractérisation pédologique, dix profils ont été réalisés: trois au niveau des Niayes de Pikine et sept au niveau des Niayes de Saint Louis.

Les échantillons de sols prélevés sur le terrain sont d'abord séchés à l'air libre pendant 10 à 15 jours, puis tamisés à $2 \mathrm{~mm}$ afin d'éliminer les particules grossières. Les fractions récupérées sont conservées dans des bocaux sur lesquels les références des échantillons sont mentionnées.

Les analyses physico-chimiques des sols ont été réalisées sur des échantillons composites à l'Institut National de Pédologie (INP) du Sénégal. Les méthodes d'analyses ainsi que les formules de calcul utilisées ont été établies par Bocoum (2004). Elles portent sur le pH, la Conductivité électrique (CE), la granulométrie, les bases échangeables (essentiellement $\mathrm{Ca}^{2+}, \mathrm{Na}^{+}, \mathrm{Mg}^{2+}, \mathrm{K}^{+}$), la capacité d'échange cationique (CEC), le carbone organique et l'azote total. La matière organique (MO) et le rapport $\mathrm{C} / \mathrm{N}$ ont été déduits par calcul.

La mesure du $\mathrm{pH}$ et celle de la conductivité électrique $(\mathrm{CE})$ ont été réalisées à partir de suspensions de sol avec des rapports sol-eau respectifs de $2 / 5$ et $1 / 10$. Par rapport à ces paramètres, les interprétations ont été effectuées respectivement en utilisant les Tableaux 2 et 3 . La granulométrie est déterminée par la méthode de tamisage, les bases échangeables par la méthode d'acétate d'ammonium, la capacité d'échange cationique (CEC) par la méthode de saturation 
au $\mathrm{NH}_{4}^{+}$, le carbone organique par la méthode de Anne et l'azote par la méthode de Kjeldahl.

\section{Traitement des données}

Le tableau de contingence portant en ligne les mesures des paramètres physico-chimiques et en colonne les dix sites secondaires correspondants aux profils pédologiques décrits (Tableau1), a été soumis à une analyse factorielle de correspondance (AFC) afin de voir les ressemblances ou similitudes entre les différents profils décrits (Cibois, 2006).

\section{RESULTATS}

Les résultats de l'AFC concernent la matrice de 10 profils et 11 paramètres physico-chimiques. Les deux premiers axes apportent à eux seuls $98 \%$ de l'information totale. L'expérience montre que le plus souvent, l'essentiel de l'information de l'AFC est fourni par ces deux axes factoriels $F_{1}$ et $F_{2}$ (Dervin, 1990). Ils définissent le plan principal $F_{1} \times F_{2}$ que nous avons choisi pour établir notre analyse. Cette analyse a conduit à une représentation simple en deux dimensions (Figure 4).

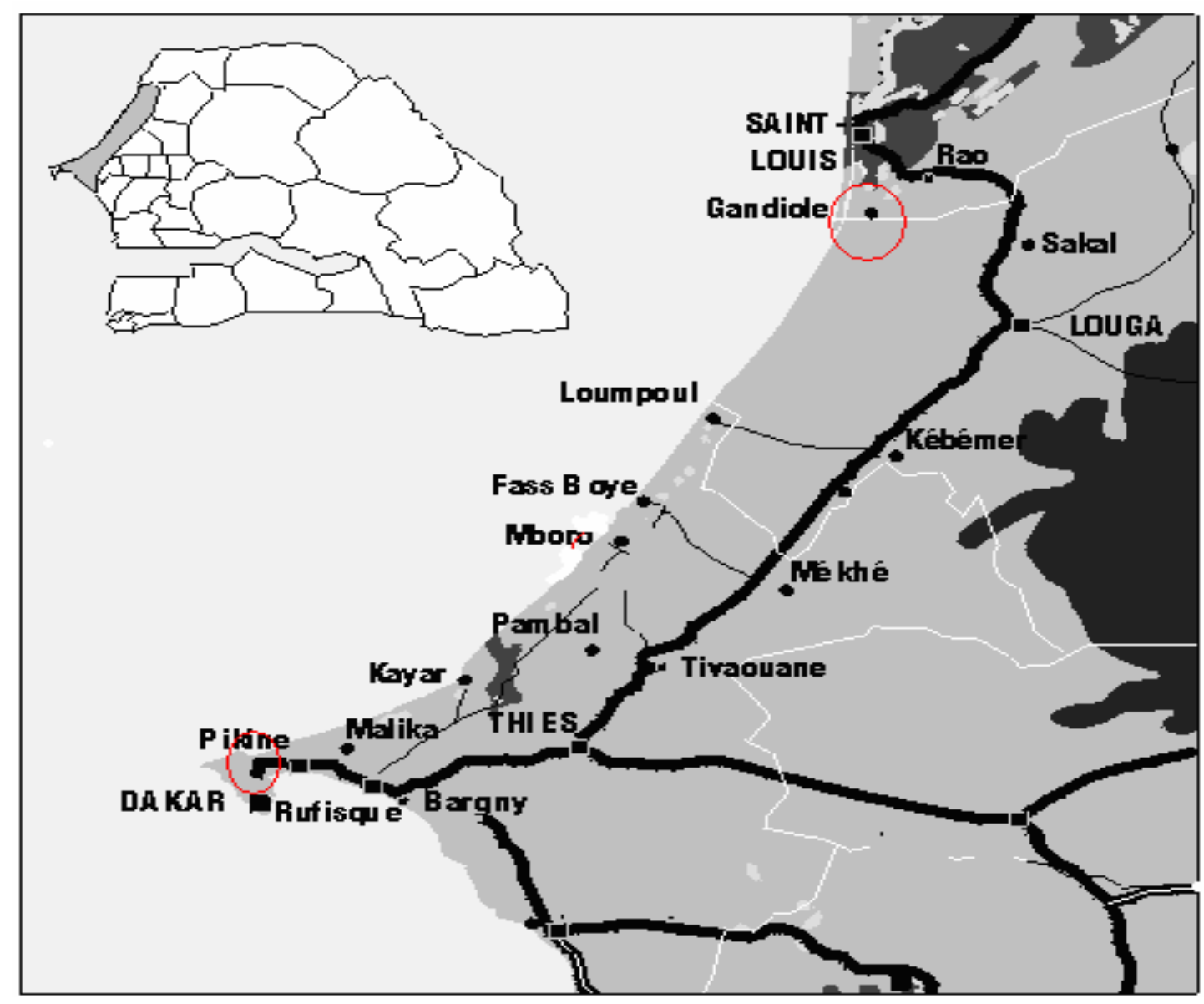

Figure 1 : Situation géographique de la zone des Niayes (Fall et al., 2001). 

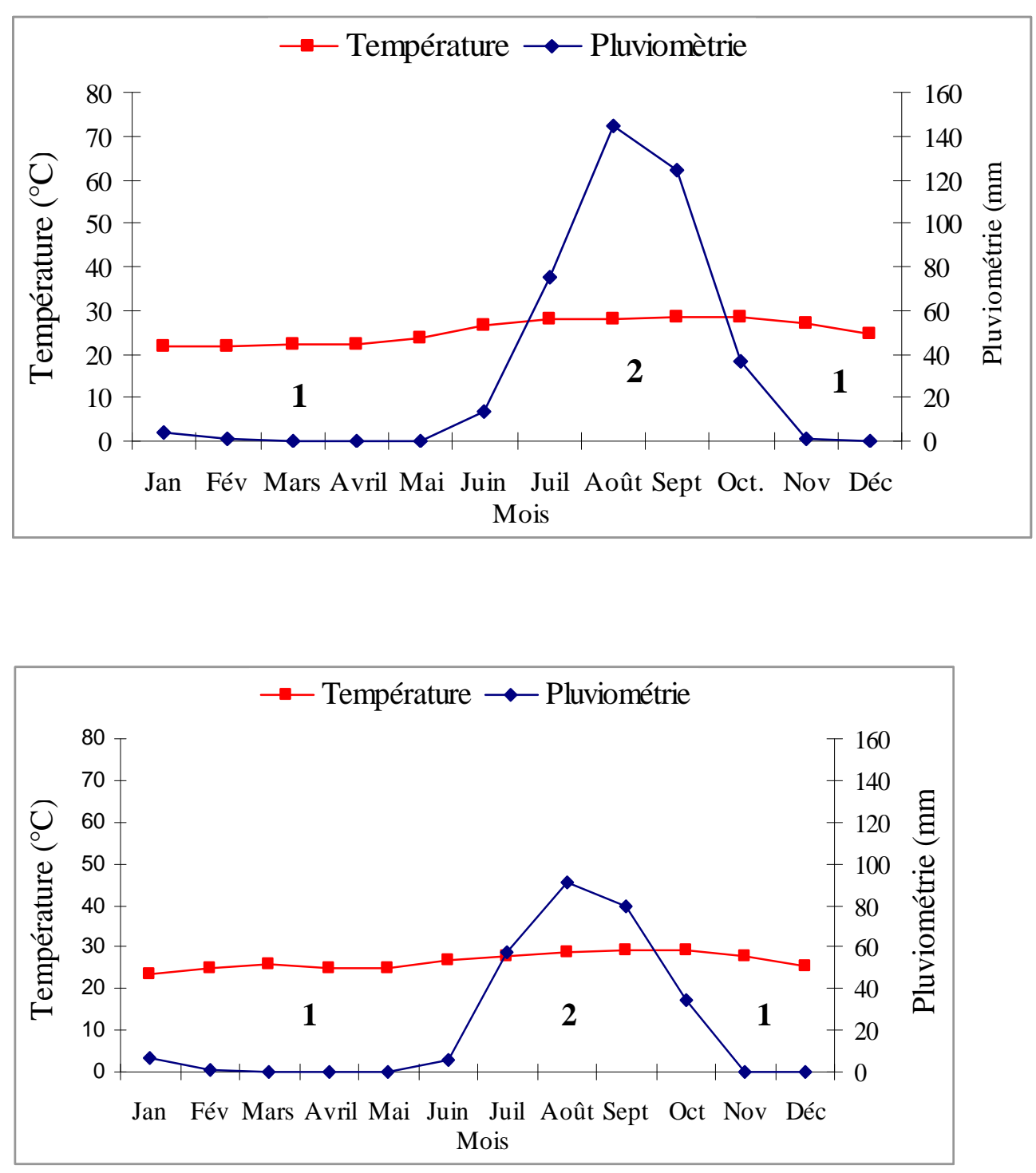

Figure 2 : Diagrammes ombro-thermiques des Niayes de Pikine (A) et de Saint-Louis (B) au cours des dix (10) dernières années : $(1)=$ saison sèche et $(2)=$ saison des pluies.

Ainsi, l'axe $\mathrm{F}_{1}$ horizontal sépare nettement le profil décrit au niveau d'un bas fond du site de Pikine Nord (Pnb) dans les abscisses positives et les autres profils en abscisses négatives. Le profil Pnb se caractérise par un sol extrêmement salin par opposition aux autres qui sont marqués par des sols légèrement ou non salins. Cet axe pourrait représenter un gradient de salinité.
Quant à l'axe $F_{2}$ vertical, il oppose deux groupes de profils tous de la Niaye de Saint Louis: ceux des sites Ricotte (Ric), Rimbakh (Rim) et Deggu Niaye (Dn) dans les ordonnées positives avec ceux des sites de Kalassane (Ka), Guinaw rail (Gr), Keur Saér (Ks) et M'boltine (Mb) dans les ordonnées négatives. Le premier groupe correspond aux profils décrits au niveau du littoral et qui se caractérisent par un sol alcalin avec un taux 


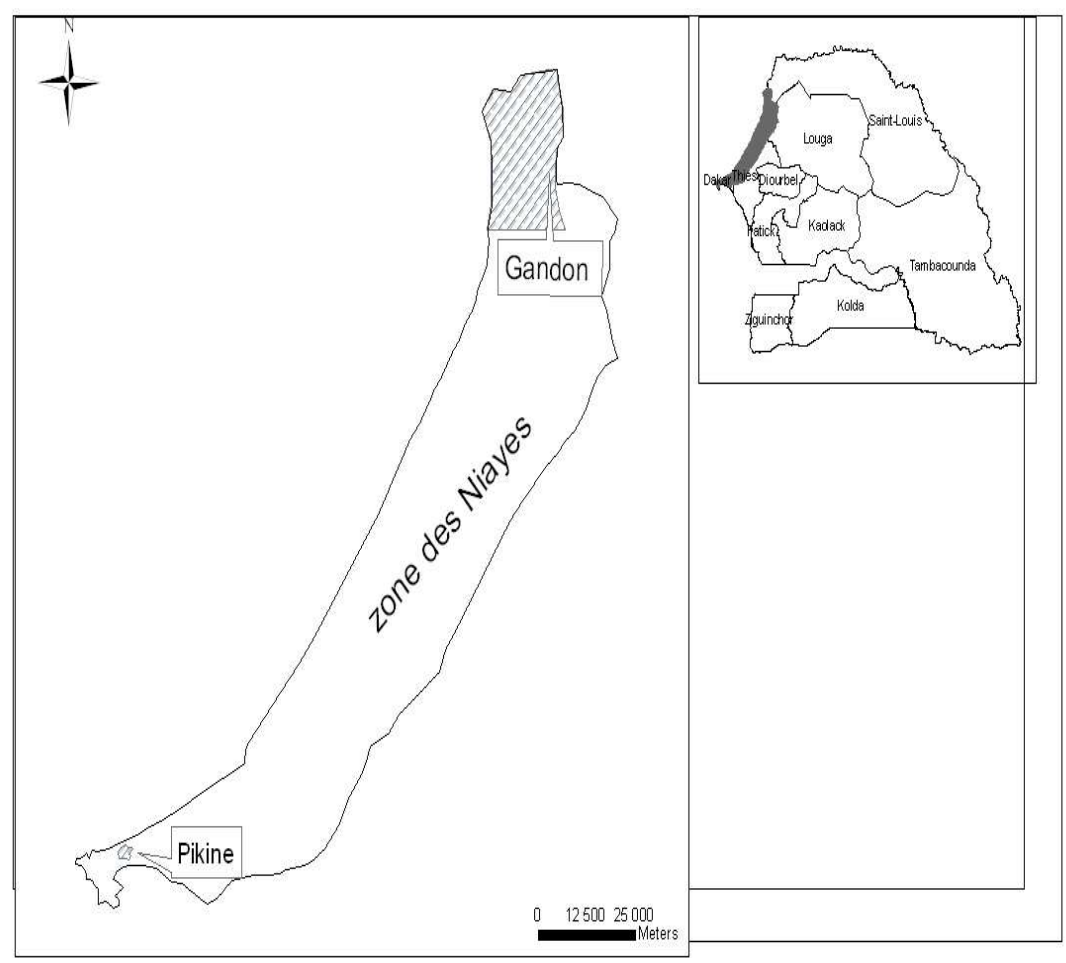

Figure 3 : Localisation des sites d'études dans les Niayes (Ndiaye, 2009).

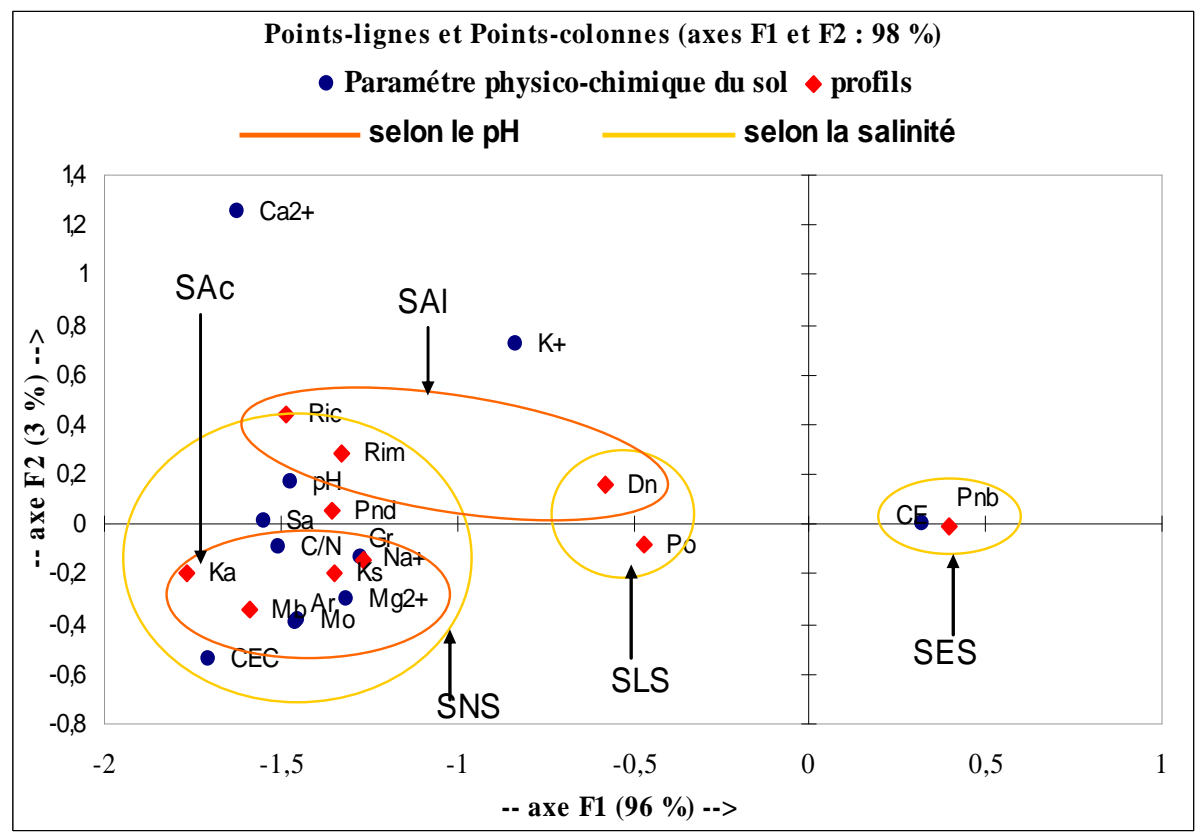

Figure 4 : Diagramme de la matrice profils / paramètres physico-chimiques des sols dans les Niayes. SAc $=$ sols acides, SAl $=$ sols alcalins, SNS = sols non salins, $\mathrm{SLS}=$ sols légèrement salins, $\mathrm{SES}=$ sols extrêmement salins. 
O. NDIAYE et al. / Int. J. Biol. Chem. Sci. 6(1): 519-528, 2012

Tableau 1 : Tableau de contingence de 10 profils pédologiques (sites) et de 11 paramètres physico-chimiques des sols.

\begin{tabular}{|c|c|c|c|c|c|c|c|c|c|c|c|}
\hline & Sites & Kalassane & Keursaér & Guinaw rail & Mboltine & $\begin{array}{c}\text { Deggu } \\
\text { niaye }\end{array}$ & Rimbakh & Ricotte & $\begin{array}{l}\text { Patte } \\
\text { d'Oie }\end{array}$ & Dune Pikine nord & $\begin{array}{c}\text { Bas fond } \\
\text { Pikine nord }\end{array}$ \\
\hline Paramètres & codes & $\mathrm{Ka}$ & $\mathrm{Ks}$ & $\mathrm{Gr}$ & $\mathrm{Mb}$ & Dn & Rim & Ric & Po & Pnd & Pnb \\
\hline Argile + limon $(\%)$ & $\mathrm{Al}$ & 8,428 & 8,652 & 8,984 & 8,875 & 7,152 & 7,06 & 2,882 & 8,083 & 7,37 & 10,22 \\
\hline Sables $(\%)$ & $\mathrm{Sa}$ & 91,572 & 91,348 & 91,016 & 91,125 & 92,847 & 92,94 & 97,118 & 91,916 & 92,63 & 89,78 \\
\hline matière organique $(\%)$ & Mo & 0,598 & 0,712 & 0,594 & 1,1 & 0,62 & 0,722 & 0,274 & 0,68 & 0,738 & 1,01 \\
\hline rapport $\mathrm{C} / \mathrm{N}$ & $\mathrm{C} / \mathrm{N}$ & 10,398 & 11,088 & 13,096 & 13,8 & 12,617 & 9,265 & 11,488 & 10,1425 & 12,19 & 13,776 \\
\hline $\mathrm{CEC} / 100 \mathrm{~g}(\mathrm{meq} / 100 \mathrm{~g})$ & CEC & 6,92 & 6,1 & 5,42 & 12,8 & 5,725 & 3,25 & 6,24 & 9,275 & 2,58 & 1,633 \\
\hline $\mathrm{Ca} 2+($ méq/100g) & $\mathrm{Ca} 2+$ & 2,125 & 1,225 & 1,9 & 2,531 & 8,812 & 7,25 & 9,975 & 2,843 & 3,96 & 1,208 \\
\hline Mg2+ (méq/100g) & $\mathrm{Mg} 2+$ & 0,425 & 1,2 & 0,925 & 2,156 & 0,968 & 0,687 & 0,775 & 1,406 & 2,55 & 2,666 \\
\hline $\mathrm{K}+($ méq $/ 100 \mathrm{~g})$ & $\mathrm{K}+$ & 0,008 & 0,011 & 0,02 & 0,03 & 0,08 & 0,372 & 0,013 & 0,166 & 0,051 & 0,379 \\
\hline $\mathrm{Na}+($ méq/100g) & $\mathrm{Na}+$ & 0,012 & 0,012 & 0,042 & 0,007 & 0,023 & 0,01 & 0,0098 & 0,023 & 0,023 & 0,032 \\
\hline $\mathrm{CE}(\mathrm{mS} / \mathrm{cm} 1 / 10)$ & $\mathrm{CE}$ & 24,45 & 59,46 & 69,8 & 41,86 & 215,65 & 62,65 & 50,86 & 246,775 & 59,66 & 5515,333 \\
\hline pH eau $(1 / 2,5)$ & $\mathrm{pH}$ & 5,768 & 6,552 & 6,648 & 6,127 & 8,195 & 7,607 & 8,172 & 7,477 & 7,7 & 8,313 \\
\hline
\end{tabular}

Tableau 2 : Appréciation de l'acidité ou de l'alcalinité du sol selon le pH (Bocoum, 2004).

\begin{tabular}{lc}
\hline Gammes de pH & Sol \\
\hline$<4,5$ & Extrêmement acide \\
$4,6-5,2$ & Très acide \\
$5,3-5,5$ & Acide \\
$5,6-6,0$ & Modérément acide \\
$6,1-6,6$ & Légèrement acide \\
$6,7-7,2$ & Neutre \\
$7,3-7,9$ & Légèrement alcalin \\
$8,0-8,5$ & Alcalin \\
$>8,6$ & Très alcalin \\
\hline
\end{tabular}


Tableau 3 : Appréciation de la salinité du sol selon la conductivité électrique (Bocoum, 2004).

\begin{tabular}{lc}
\hline Conductivité Electrique/10 $(\boldsymbol{\mu m h o s})$ & Sol \\
\hline$<250$ & Non salin \\
$250-500$ & Légèrement salin \\
$500-1000$ & Salin \\
$1000-2000$ & Très salin \\
$>2000$ & Extrêmement salin \\
\hline
\end{tabular}

assez élevé de calcium $\left(\mathrm{Ca}^{2+}\right)$. Par contre, le second groupe est constitué de profils réalisés dans des milieux plus ou moins éloignés du littoral et qui sont constitués de sols modérément à légèrement acides avec des teneurs en calcium beaucoup moins importantes. Donc l'axe vertical $\mathrm{F}_{2}$ définirait un gradient de proximité de l'océan en relation avec l'alcalinité ou l'acidité et la teneur en calcium.

\section{DISCUSSION}

La zone des Niayes présente une grande diversité de type de sols. Ainsi, en considérant la salinité, l'analyse factorielle de correspondance révèle l'existence de trois entités de sols: les sols extrêmement salins (SES), rencontrés uniquement dans les bas fonds de la Niaye de Pikine; les sols légèrement salins (SLS), qu'on retrouve aussi bien dans les pentes de la Niaye de Pikine que les bas fonds proches de l'océan de la Niaye de Saint Louis et les sols non salins (SNS), dans les sites de la Niaye de Saint Louis plus ou moins éloignés de la mer et les zones dunaires de la Niaye de Pikine (Figure 4).

La salinisation des sols au niveau de la Niaye de Pikine pourrait être en majeure partie attribuée à un déficit pluviométrique qui est à l'origine de l'abaissement de la nappe phréatique et à l'intrusion des eaux de mer à l'intérieur des terres. Ce déficit s'illustre par une réduction de la saison pluvieuse concentrée sur 3 à 4 mois au lieu de 5 à 6 mois auparavant (Faye et al., 1995). Selon cet auteur, la pluviométrie annuelle dans les Niayes, qui était en moyenne de $684 \mathrm{~mm}$ de 1931 à 1960 est tombée à $480 \mathrm{~mm}$ de 1961 à 1990. Une analyse récente a montré que cette moyenne est de $271 \mathrm{~mm}$ durant la période 1991 à 2009 (Ndiaye, 2009).

Ce phénomène de salinisation des sols est plus marqué dans les bas fonds où la nappe phréatique est à faible profondeur. Cette salinisation par endroit, serait à l'origine de la disparition de nombreuses espèces végétales (Diallo et al., 2009) dans la zone des Niayes.

Le phénomène d'invasion marine, qui peut s'étendre sur plusieurs kilomètres à l'intérieur des terres est un grand risque pour les régions côtières tributaires des eaux souterraines pour leur approvisionnement en eau (Morsli, 2007). Sous certaines conditions, l'eau salée se propage à l'intérieur des terres et contamine les eaux de la nappe située à proximité de la mer. Cette invasion des eaux douces par les eaux salées a pour effet une dégradation des sols et leur salinisation.

Quant au littoral de la Niaye de Saint Louis, la salinisation moins marquée des sols pourrait s'expliquer en plus des irrigations avec les eaux salées des nappes souterraines, par les apports non négligeables des embruns marins (Diakhaté, 1986). Ces derniers peuvent être restitués facilement à la nappe par les pluies dans le cas où le sol est très perméable, comme ils peuvent être fixés par le sol dans le cas où celui-ci présente une mauvaise perméabilité (Morsli, 2007) provoquant ainsi la salinisation des sols atteints. Par contre, Douaoui et Hartani (2007) ont montré que l'utilisation des eaux souterraines salines pour l'irrigation contribue à la dégradation de la qualité des sols qui se manifeste par une augmentation de la salinisation ou une sodisation. La proximité de la mer a également une influence sur la salinité des 
sols par l'intermédiaire des pluies chargées de chlorure (Margat, 1961).

Les facteurs explicatifs de la salinisation des nappes sont multiples dans cette partie, il s'agit de la sécheresse, la position estuarienne de la zone du Gandiolais (Gandon), les effets du barrage de Diama et le canal de délestage ouvert sur la Langue de Barbarie à Saint Louis (Thiam, 2005).

Cependant, en tenant compte du potentiel en hydrogène et de la teneur en calcium des sols, nous avons identifié deux groupes de sols: les sols alcalins ( $\mathrm{SAl}$ ), avec un taux assez important de calcium qu'on retrouve uniquement dans les sites de la Niaye de Saint Louis proches de l'océan, et les sols modérément à légèrement acides (SAc), caractérisés par des teneurs moins importantes en calcium qui caractérisent les sites de la Niaye de Saint Louis assez éloignés de la mer (Figure 4).

Au niveau des Niayes de Saint Louis, nous avons noté une nette concordance entre le $\mathrm{pH}$ et la teneur en calcium échangeable des sols. En effet, les variations de concentrations en calcium échangeables sont à relier au $\mathrm{pH}$. Lorsque ce dernier est supérieur à 7 , le calcium occupe au moins les deux tiers des cations échangeables (Pernes-Debuyser et Tessier, 2002).

Selon certains auteurs, l'alcalinité des sols est généralement due à l'abondance des ions sodium et calcium plus importants en milieu littoral. L'alcalinisation peut dans ce cas se manifester sur une zone aménagée et irriguée par l'homme en quelques dizaines d'années, voire quelques années, affectant considérablement les propriétés physiques et hydriques des sols (Bardiero et al., 1995).

\section{Conclusion}

Cette étude a permis de distinguer dans les Niayes de Saint-Louis du point de vue agro-pédologique, deux sous zones distinctes: la Niaye continentale caractérisée par des sols légèrement acides à faible teneur en calcium, ne recevant aucune influence marine et la Niaye littorale très influencée par la mer, sensible à l'intrusion marine, marquée par des sols alcalins riches en calcium parfois légèrement salés.

D'une manière générale, on peut distinguer quatre aspects importants sur la pédologie dans la zone des Niayes: une salinisation plus marquée dans la partie méridionale, une alcalinisation corrélée à l'abondance du calcium plus importante dans la Niaye littorale de Saint-Louis, une acidification uniquement notée au nord dans la partie continentale et un ensablement des cuvettes maraîchères dans la quasi-totalité de la zone.

Ces quatre facteurs, souvent limitant, sont assez bien représentés, selon l'humidité des sols, elle même fonction de la topographie, et des précipitations.

\section{REFERENCES}

Anonyme. 1990. Inventaire biophysique de la région des Niayes. Conservation des terroirs du littoral. Direction des Eaux et Forêts et Chasses, Dakar-Sénégal, 262 p.

Ba M. 2008. Agriculture et Sécurité Alimentaire. CIRAD ; p 59.

Barbiero L, Valles V, Regeard A. 1995. Précipitation de la fluorine et contrôle géochimique du calcium dans des sols alcalins du Niger. Conséquences pour une estimation quantitative de l'évolution géochimique des sols. C.R. Acad. Sci., 321(série lia) : 1147-1154.

Bocoum M. 2004. Méthodes d'analyses des sols. Doc de travail. Institut National de Pédologie, Dakar-Sénégal, 55p.

Cibois P. 2006. Principe de l'Analyse Factorielle. 34 p. http://cibois. pagesperso-orange.fr/PrincipeAnalyseFac torielle.pdf

Dervin C. 1990. Comment Interpréter les Résultats d'une Analyse Factorielle de Correspondances? Mathématiques Informatique - Biométrie. I.N.R.A I.N.A.P.G : Paris ; 77 p.

Douaoui AT, Hartani T. 2007. Impact de l'irrigation par les eaux souterraines sur la dégradation des sols de la plaine du BasChéliff, Algérie. Actes du troisième 
atelier régional du projet Sirma, Nabeul, Tunisie, $5 \mathrm{p}$.

Dia B. 1992. La fixation des dunes au Sénégal. Projet de Conservation des Terroirs du Littoral Nord. Gestions des ressources côtières et littorales du Sénégal. Direction des eaux et Forêts et Chasses, Dakar-Sénégal, 201 p.

Diakhate M. 1986. Le barrage de Diama: essai sur l'évaluation de ses impacts potentiels. Revue de Géographie de Lyon, 61(1): 4361.

Diallo A, Guisse A, Faye M, Saradoum G. 2009. Variabilité floristique de la végétation herbacée de la Niaye de Pikine au Sénégal. Rev. Ecol. Terre Vie, 64: 123133.

Diallo A. 2006. Etude de la variation spatiale de la végétation herbacée des Niayes de la région de Dakar par une approche phytosociologique. Mémoire de DEA, UCAD, 72 p.

Fall AS, Fall ST, Cisse I, Badiane AN, Diao MB, Fall CA. 2001. Caractérisation de la zone des Niayes. In CITES Horticoles en Sursis? L'Agriculture Urbaine les Grandes Niayes au Sénégal. IDRC. http//idrc.ca/en/ev-27906-201-1

DO_TOPIC.htm.

Faye O, Gaye O, Fontenille D, Hébrard G, Konate L, Sy N, Hervé JP, Touré Y, Diallo S, Molez JF, Mouchet J. 1995. La sécheresse et la baisse du paludisme dans les Niayes du Sénégal. Cahiers de Santé 5. ORSTOM: 299-305.

Khouma M. 1998. Les grands types de sols au Sénégal. Rapport sur les sols du monde, ISRA CNRA de Bambey, $21 \mathrm{p}$.

Margat J. 1961. Les eaux salées au Maroc: hydrogéologie et hydrochimie. Notes et Mem. Serv. Géol. Mar., 151: 65-66.

Morsli B. 2007. Etude de l'intrusion marine et de ses répercussions sur la dégradation des sols: cas des zones côtières d'Alger Est. Actes des JSIRAUF, Hamoi, 6 p.

Ndiaye O. 2009. Contribution à la caractérisation du sol et de la végétation des Niayes de Pikine et de Saint Louis au Sénégal. Mém de DEA, UCAD, 59 p.

Pernes-Debuyser A, Tessier D. 2002. Influence du $\mathrm{pH}$ sur les propriétés des sols: l'essai de longue durée des 42 parcelles à Versailles. Rev. Sci. Eau, 15( $\mathrm{n}^{\circ}$ spécial): 27-39.

Thiam EI. 2005. La problématique des eaux douces dans l'estuaire du fleuve Sénégal: l'exemple de la zone du Gandiolais. Mém de DEA, UCAD, 56p.

Trochain JL. 1940. Contribution à l'étude de la végétation du Sénégal. Mem. IFAN, 1(2): 433. 